\title{
Integrable structures in LGTs near the deconfinement transition
}

\section{Caselle, F. Gliozzi}

Dipartimento di Fisica Teorica dell'Università di Torino and INFN sezione di Torino, via P. Giuria 1, I-10125, Torino, Italy

E-mail: caselle, gliozzi@to.infn.it

\section{G. Delfino}

International School for Advanced Studies (SISSA)

via Beirut 2-4, I-34014 Trieste, Italy and INFN sezione di Trieste

E-mail: delfinoesissa.it

\section{P. Giudice}

School of Mathematics, Hamilton Building Trinity College Dublin 2, Ireland

E-mail: giudice@maths.tcd.ie

\section{P. Grinza*}

Departamento de Física de Particulas and IGFAE,

Universidad de Santiago de Compostela

E-mail: pgrinza.grinza@usc.es

\section{S. Lottini}

Westfälische Wilhelms-Universität Münster,

Wilhelm-Klemm-Strasse 9, 48149 Münster, Germany

E-mail: s.lottini@uni-muenster.de

\section{N. Magnoli}

Dipartimento di Fisica, Università di Genova and INFN sezione di Genova, via Dodecaneso 33, I-16146, Genova, Italy

E-mail: magnoli@ge.infn.it

In this contribution we review some recent results about the emergence of $2 \mathrm{D}$ integrable systems in 3D Lattice Gauge Theories near the deconfinement transition. We focus on some concrete examples involving the flux tube thickness, the ratio of k-string tensions and Polyakov loops correlators in various models.

The XXVII International Symposium on Lattice Field Theory - LAT2009

July 26-31 2009

Peking University, Beijing, China

\footnotetext{
${ }^{*}$ Speaker.
} 


\section{Introduction}

The proposal of the Svetistky-Yaffe conjecture [1] in the early-80's came as a new interesting tool to both understand the physics of the confining/deconfining transition, and a smart way-out to circumvent the problem of slow Monte Carlo simulations in Lattice Gauge Theories (LGT).

In brief, the conjecture establishes a relation between the deconfining phase transition of a LGT with gauge group $G$ in $(d+1)$-dimensions and the symmetry breaking phase transition of a given spin model in $(d)$-dimensions with symmetry group $\mathscr{C}_{G}$, i.e. the center of the group $G$. In particular, in the case of continuous phase transitions a standard renormalisation group analysis shows that the critical behaviour of the two models must be the same, i.e. they belong to the same universality class. Hence, universal quantities like critical exponents and amplitude ratios can be (more easily) calculated in the spin model and then extended to the LGT. Another key feature of the conjecture is the possibility to establish a dictionary between the observables of the LGT and the corresponding ones in the spin model.

After more than twenty years, the Svetistky-Yaffe conjecture is considered a reliable tool to study a LGT in the neighbourhood of the deconfinement transition. As a step forward, one can try to take advantage of the conjecture, and get a deeper quantitative understanding of a LGT in the neighbourhood of the deconfining transition. From this point of view, three-dimensional LGT offers a unique opportunity, because they correspond to two-dimensional spin models. There are diverse motivations making such a class of spin models a privileged one.

On the one hand, they are exactly solved at the critical point, i.e. where they are relevant for the analysis of the LGT. In fact, it was shown that a large class of spin models displaying a symmetry breaking phase transition allows for an exact solution in terms of two-dimensional Conformal Field Theories (CFT) [2]. What makes the two-dimensional case special is the fact that the conformal algebra becomes infinite-dimensional, and as a consequence the corresponding theory is much more constrained that in other dimensions. Such a richness in structure reflects at the quantitative level too. In fact, it is possible to give a complete classification of the space of fields of a given CFT together with their exact scaling dimension. The most direct implication of this fact is the exact calculation of all the critical exponents of the corresponding phase transition. Another interesting feature is given by the possibility to calculate any $n$-point correlation function among the fields of the theory, as solutions of some well established differential equations. In the perspective of the S-Y conjecture, such host of exact results can be directly extended to the corresponding LGT, giving a complete characterisation of the universality class of the deconfinement transition.

On the other hand, the S-Y conjecture can be used to give an effective description of the LGT, by means of the corresponding spin model, not only at the critical point but also in the scaling region. The first non-trivial step is to show that the plaquette operator of the LGT is mapped into a mixture of the energy and identity operators [3]. Then, it is possible to show that the scaling region of the LGT corresponds to the thermal perturbation of the CFT describing the critical point of the spin model.

This aspect has important consequences, because it happens that such QFTs turn out to be integrable for most of the cases of interest in the present context. Integrability means that an infinite number of integrals of motion exists. The main consequence in $(1+1)$ dimensions is the fact that the scattering theory is very constrained, because the $S$-matrix is factorised in products of two-body 
interactions, and inelastic processes are forbidden. These facts allow to write down the so-called Yang-Baxter equations for the 2-particle $S$-matrix. Then, such an $S$-matrix can be computed exactly by imposing the previous equations and the usual requirements of unitarity and crossing (for a review about Integrable QFTs see [4]). An obvious consequence is that also the spectrum of the masses of the bound states of the theory is known exactly, since they are represented by the simple poles of the $S$-matrix in the physical strip.

Another useful aspect of dealing with an integrable theory is the possibility to use the spectral expansion (form factors can be computed exactly in integrable QFTs) for correlation functions. Such a fact gives the possibility to describe with a good deal of accuracy the large distance behaviour of the correlation functions. As we will see in the following, this is a key point in our approach.

In the present contribution we gathered our main results obtained in several models, and with different types of observables. In sect. 2 we report on the study of the baryon (three-quark) potential in the 3D SU(3) LGT using the three-spin correlation function of the 2D 3-state Potts model [5]. Sect. 3 is devoted to the study of the behaviour of the flux tube thickness in the 2D SU(2) LGT via the corresponding 2D Ising model [9]. Finally, in sect. 4 we report on the exact calculation of the ratio of k-string tensions in the $3 \mathrm{D} Z_{4}$ LGT my means of the mapping on the $2 \mathrm{D}$ Sine-gordon model [14].

\section{Three-quark potential [5]}

In these last years much interest has been attracted by the study of the three-quark potential in LGT. Besides the obvious phenomenological interest of the problem, the three-quark potential is also a perfect tool for testing our understanding of the flux tube model of confinement and of its theoretical description in terms of effective string models. Thanks to the improvement in lattice simulations (a summary of numerical results can be found in [6]), the qualitative behaviour of the three-quark potential is now rather well understood (for a recent review see [7]). Let us briefly review the key points.

For large interquark distances the three-quark potential is well described by the so-called $\mathbf{Y}$ law which assumes a flux tube configuration composed by three strings which originate from the three quarks and join in the Steiner point which has the property of minimising the overall length of the three strings. This picture is also in agreement with what one would naively find using standard strong coupling expansion. Notice however that due to the roughening transition this is only a qualitative indication, and cannot be advocated as a "proof" of the $\mathbf{Y}$ law.

At shorter distances a smooth crossover toward the so called $\Delta$ law is observed. According to the $\Delta$ law the three-quark potential is well approximated by the sum of the three two-quark interactions. More precisely the $\Delta$ law assumes that the three-quark correlator (let us call it $G_{3}\left(x_{1}, x_{2}, x_{3}\right)$ where $x_{j}$ denotes the position of the $j^{\text {th }}$ quark) is related to the quark-antiquark correlator $G_{2}\left(x_{i}, x_{j}\right)$ as follows:

$$
G_{3}\left(x_{1}, x_{2}, x_{3}\right) \sim \sqrt{G_{2}\left(x_{1}, x_{2}\right) G_{2}\left(x_{2}, x_{3}\right) G_{2}\left(x_{1}, x_{3}\right)}
$$

thus leading to a potential which increases linearly with the sum of the three interquark distances. The scale where the transition between these two behaviours seems to occur, according to the most recent simulations, is around $0.8 \mathrm{fm}$. 
To improve our understanding of the baryon states, it would be important now to have some quantitative insight in the above described picture, as well as to have some theoretical argument to explain why instead of having a single shape stable for all the interquark distances a $\Delta \rightarrow Y$ crossover occurs. Moreover, since the crossover region happens to occur exactly in the range of distances which is interesting from a phenomenological point of view, it would be important to have some kind of theoretical description of this crossover with which to compare the numerical data.

In this respect the present study of the three-point function in the $2 \mathrm{~d} \mathbb{Z}_{3}$ Potts model is a perfect laboratory to address this problem. Besides the obvious similarity of the two settings it is also possible to find a direct relation, since by the Svetitsky-Yaffe conjecture the behaviour at high temperature of the three-quark correlator for a $S U(3)$ or a $\mathbb{Z}_{3}$ gauge model in $(2+1)$ dimensions is mapped into the behaviour of the $2 \mathrm{~d} \mathbb{Z}_{3}$ Potts three-point function, in analogy to what happens for the quark-antiquark potential which is mapped onto the $\langle\sigma \bar{\sigma}\rangle$ correlator.

In the large distance regime, the form factor approach (spectral expansion) plays a central role due to the integrability of the theory. The appearance of the Steiner point is quite natural in such a framework (even if completely non-trivial), and it is strongly related to the integrable structure of the model. Here we quote the final result (leading and sub-leading orders) in the case of an equilateral triangle of side $R$ (see [5] for details)

$$
\begin{aligned}
G^{(3)}\left(x_{1}, x_{2}, x_{3}\right) \simeq & \frac{\left(F_{\bar{A}}^{\sigma}\right)^{3} \Gamma_{A A}^{\bar{A}}}{\pi} K_{0}(\sqrt{3} m R)+ \\
& +6\left(F_{\bar{A}}^{\sigma}\right)^{2} \int_{-\infty}^{\infty} \frac{\mathrm{d} \theta}{(2 \pi)^{2}} F_{A A}^{\sigma}(\theta+i \pi / 3) K_{0}\left(2 m R \cosh \frac{\theta}{2}\right)
\end{aligned}
$$

where $r_{Y}=\sqrt{3} R$ is the minimal distance given by the emergence of the Steiner point. On the other hand, the short distance regime is studied by means of the so-called Conformal Perturbation Theory (CPT) [8], which is a perturbative approach built upon the exact CFT solution of the model at the critical point (integrability is not needed here). We first notice that, at criticality, the $\Delta$ law is exact. In fact we see that the relation (2.1) is fulfilled exactly by 2- and 3-p correlator functions of a given CFT. Hence, we used the CPT framework to compute the corrections induced by the thermal perturbation, and we are able to explicitly compute the perturbative corrections to the $\Delta$ law.

From a physical point of view the scenario which emerges is a smooth crossover, as the distance among the three points increases, from a short distance behaviour in which the three point function is dominated by the three spin-spin interactions along the edges of the triangle to a large distance behaviour in which the strong coupling expectation (the three spins joined by a path of minimal length) is fully realised.

This scenario is confirmed by the numerical simulations which turn out to be in remarkable agreement with our theoretical results (see figures 1,2). Indeed the important consequence of having exact analytic results for the two expansions is that we are able to compare our predictions with triangles of any size, both smaller and larger than the correlation length.

In conclusion, we would like to stress that the S-Y conjecture allowed us to check with some solid field-theoretic argument that, at least near the deconfinement temperature, a crossover between $\mathbf{Y}$ and $\Delta$ laws occurs at some intermediate scales. 


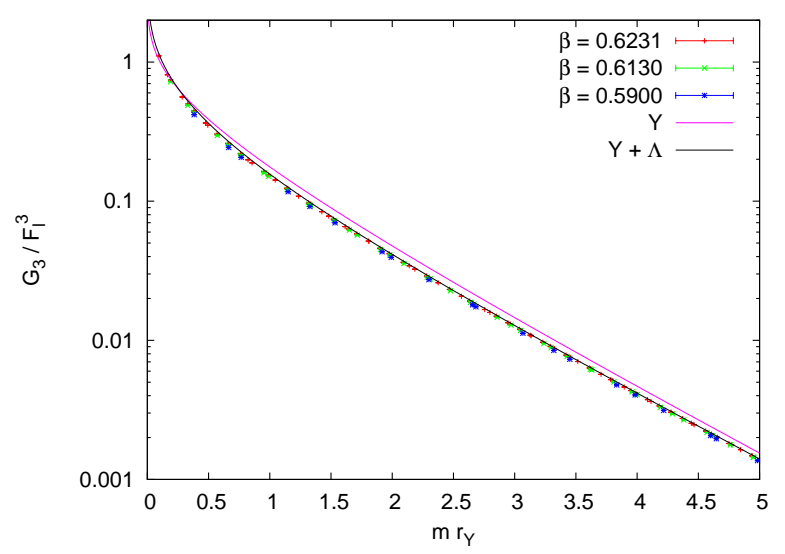

Figure 1: Three-point function (suitably normalised, see [5] for details): spectral expansion (form factors), and Monte Carlo data.

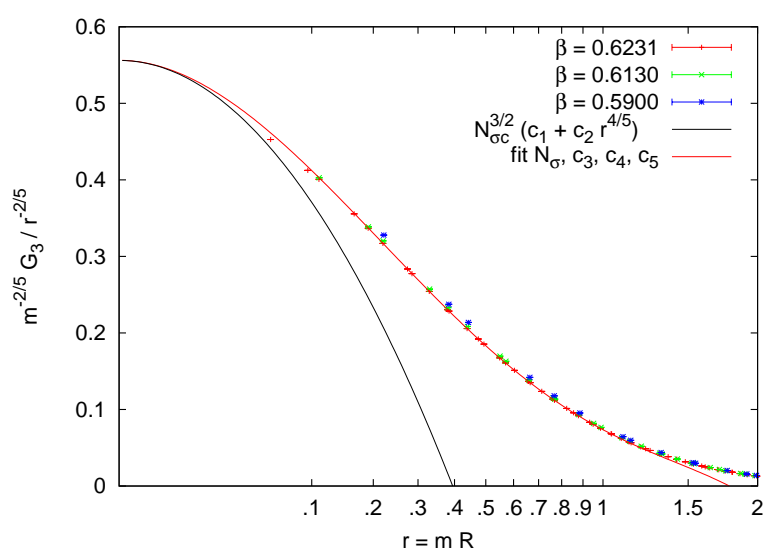

Figure 2: Three-point function (suitably normalised, see [5] for details): short-distance expansion (Conformal Perturbation Theory), and Monte Carlo data.

\section{Flux tube thickness [9]}

The distinctive feature of the interquark potential in a confining gauge theory is that the colour flux is confined into a thin flux tube, joining the quark-antiquark pair. As it is well known the quantum fluctuations of this flux tube, which are assumed to be described by a suitable effective string model, lead to a logarithmic increase of the width of the flux tube as a function of the interquark distance $R$. This behaviour was discussed many years ago by Lüscher, Münster and Weisz in [10] and is one of the most stringent predictions of the effective string description of confining LGTs.

A natural question is what happens of this picture at the deconfinement point. One would naively expect a sudden jump of the flux tube thickness from a log to a linear dependence from the interquark distance. However we shall show in this work that this is a misleading picture.

A tentative answer to this question can be obtained in the effective string framework. By using a duality transformation it is possible to show that as the temperature increases the log behaviour smoothly moves to a linear behaviour, thus excluding a log to linear transition at the deconfinement 
point. The simultaneous dependence of the flux tube thickness on the two variables $R$ and $N_{t}$ can be evaluated exactly only in the gaussian limit*. For the details of the calculations we refer the reader to the paper [12].

To the purpose of the present work, we are only interested in the two asymptotic limits: large $N_{t}$ and finite $R$ (which is the zero temperature limit where we expect a log type behaviour) and the opposite one: large $R$ and small $N_{t}$ which is high temperature limit.

One finds:

$$
\begin{aligned}
& w^{2} \sim \frac{1}{2 \pi \sigma} \log \left(\frac{R}{R_{c}}\right) \quad\left(N_{t}>>R>>0\right) \\
& w^{2} \sim \frac{1}{2 \pi \sigma}\left(\frac{\pi R}{2 N_{t}}+\log \left(\frac{N_{t}}{2 \pi}\right)\right) \quad\left(R>>N_{t}\right)
\end{aligned}
$$

As it is easy to see in the second limit the logarithmic dependence is on $N_{t}$ (the inverse of the temperature) and not on $R$ which appears instead in the linear correction. However this result strongly relies on the effective string approximation (even worse on the gaussian limit of the effective string) and it would be nice to have some kind of independent evidence.

Hence, we propose an alternative way to address the above question in the vicinity of the deconfinement transition using the Svetitsky-Yaffe conjecture which is a very powerful tool to study the finite $\mathrm{T}$ behaviour of a confining LGT in the vicinity of the deconfinement point, at least for those LGTs whose deconfinement transition is of second order.

This gives us a non trivial opportunity to check the effective string predictions. If we choose a $(2+1)$ dimensional LGT with a gauge group with center $Z_{2}$ (like the gauge Ising model or the SU(2) or SP(2) LGTs which all have continuous deconfinement transitions), the target spin model is the $2 \mathrm{~d}$ Ising model in the high temperature symmetric phase for which several exact results are known. In particular we shall see that it is possible to study analytically the equivalent of the flux tube thickness. Leaving the details to [9], it is possible to show that, by means of the spectral expansion over form factors, for very large separations between the spins (quarks) the flux tube thickness in the Ising model behaves like

$$
w^{2} \simeq \frac{\pi}{2} \frac{R}{\sigma N_{t}}+\ldots, \quad N_{t} \rightarrow \beta_{c} .
$$

Remarkably enough the results that we find agree, up to non-universal constants, with the effective string ones thus strongly supporting the idea of a smooth transition from a log to a linear behaviour as the temperature increases. A numerical confirmation of such a scenario has been recently found in [13].

\section{K-string tensions ratios [14]}

In a recent paper [15], it was argued from simple scaling properties of suitable Polyakov loop correlators that the k-string tensions have the following low temperature asymptotic expansion

$$
\sigma_{k}(T)=\sigma_{k}-c \frac{\pi}{6} T^{2}+\mathscr{O}\left(T^{3}\right) ; c=(d-2) \frac{\sigma_{k}}{\sigma},
$$

* Including higher order terms in the effective string action makes the problem too difficult, even if some recent result in the framework of the covariant quantisation suggest that some simplification could occur if one chooses to study the whole Nambu-Goto action [11]. 
where $c$ is the central charge of the underlying $2 \mathrm{D}$ conformal field theory describing the IR behaviour of the k-string. As a consequence, their ratios are expected to be constant up to $T^{3}$ terms:

$$
\frac{\sigma_{k}(T)}{\sigma(T)}=\frac{\sigma_{k}}{\sigma}+\mathscr{O}\left(T^{3}\right)
$$

The low temperature data presented in support of this expectation were taken from Monte Carlo simulations on a particular system, namely a (2+1)-dimensional $\mathbb{Z}_{4}$ gauge model, which is the simplest exhibiting more than just the fundamental string.

The main conjecture we want to verify in this work is that $\sigma_{k}(T) / \sigma(T)$, at least in that $\mathbb{Z}_{4}$ gauge system, is in fact independent of the temperature in the whole of the confining regime. To check this idea, a handy fact comes useful, namely that, as the system approaches the deconfinement transition, and the string picture begins fading, another approach is made available by the Svetitsky-Yaffe (S-Y) conjecture [1], which allows to reformulate the system in a totally different perspective, based on a two-dimensional integrable theory in which, however, the near- $T_{c}$ counterpart of the low-temperature result cited above can be nicely found. It turns out that the deconfinement transition of the $3 \mathrm{D} \mathbb{Z}_{4}$ gauge model is second order and, according to the SY conjecture, belongs to the same universality class of the 2D symmetric Ashkin-Teller (AT) model. As a matter of fact, such a model possesses a whole line of critical points along which the critical exponents vary continuously. The S-Y conjecture tells us that if a $2+1$ dimensional gauge model with center $\mathbb{Z}_{4}$ displays a second-order transition, then its universality class is associated to a suitable point of the critical line of the 2D AT model [16]. For instance, it has been argued [18] that the critical 2+1 $S U$ (4) gauge theory belongs to the universality class of a special point of the AT model, known as the four-state Potts model. More generally, the class of models with gauge group $\mathbb{Z}_{4}$ depends on two coupling constants $\alpha$ and $\beta$, and the universality class of the deconfining point $P$ varies with the ratio $\alpha / \beta$.

The two-dimensional AT model can be seen in the continuum limit as a bosonic conformal field theory plus a massive perturbation (i. e. a Sine-Gordon theory) driving the system away from the critical line. Thus, a map between (a neighbourhood of) the AT critical line and the SineGordon phase space is provided. This theory is integrable, and the masses of its lightest physical states (first soliton and first breather mode, of masses $M$ and $M_{1}$ [17]) correspond to the tensions $\sigma(T)$ and $\sigma_{2}(T)$ near $T_{c}$, whose ratio can be analytically evaluated and turns out to be

$$
\lim _{T \rightarrow T_{c}} \frac{\sigma_{2}(T)}{\sigma(T)}=\frac{M_{1}}{M}=2 \sin \frac{\pi}{2}(2 v-1),
$$

where $v$ is the thermal exponent in two dimensions.

As a consequence, on the gauge side, we have two different ways to verify the conjecture. One is to directly estimate the ratio $M_{1} / M$ by measuring the Polyakov-Polyakov correlators in the two non-trivial representations of $\mathbb{Z}_{4}$ near the deconfining temperature. The other is to evaluate the thermal exponent of the gauge system at the deconfining temperature. Either method gives a value of $M_{1} / M$ which can be compared with the ratio $\sigma_{2} / \sigma$ evaluated at $T=0$. In particular, choosing $(\alpha, \beta)=(0.050,0.207)$, we obtained the following results:

- at $T=0$ we have [15]

$$
\frac{\sigma_{2}}{\sigma}=1.610(13)
$$


- at $T \rightarrow T_{c}^{-}$, the fit of the data for the Polyakov-Polyakov correlators in the fundamental and double-fundamental reps gives [14]

$$
\sigma_{2}\left(T \sim T_{c}\right) / \sigma\left(T \sim T_{c}\right)=\frac{M_{1}}{M}=1.612(46),
$$

- at $T \rightarrow T_{c}^{-}$, the evaluation of the thermal critical exponent $v$ gives [14] (the systematic error is quoted in square brackets)

$$
\sigma_{2}\left(T \sim T_{c}\right) / \sigma\left(T \sim T_{c}\right)=\frac{M_{1}}{M}=2 \sin \frac{\pi}{2}(2 v-1)=1.6124(71)[102],
$$

where we can see that the latter estimates at $T \rightarrow T_{c}^{-}$give compatible results which nicely agree with the ratio $\sigma_{2} / \sigma$ evaluated at $T=0$.

Acknowledgements The work of P.G. is partially supported by MEC-FEDER (grant FPA200801838), by the Spanish Consolider-Ingenio 2010 Programme CPAN (CSD2007-00042) and by Xunta de Galicia (Conselleria de Educacion and grant PGIDIT06PXIB296182PR).

\section{References}

[1] B. Svetitsky and L. G. Yaffe, Nucl. Phys. B 210 (1982) 423.

[2] P. Ginsparg, in "Fields, Strings and Critical Phenomena", (Les Houches, Session XLIX, 1988) ed. by E. Brézin and J. Zinn Justin, 1989.

[3] F. Gliozzi and P. Provero, Phys. Rev. D 56 (1997) 1131.

[4] G. Mussardo, Phys. Rept. 218 (1992) 215.

[5] M. Caselle, G. Delfino, P. Grinza, O. Jahn and N. Magnoli, J. Stat. Mech. 0603, P008 (2006).

[6] C. Alexandrou, P. de Forcrand and A. Tsapalis, Phys. Rev. D 65 (2002) 054503. T. T. Takahashi et al., Phys. Rev. D 65 (2002) 114509.

[7] P. de Forcrand and O. Jahn, Nucl. Phys. A 755 (2005) 475.

[8] A. B. Zamolodchikov, Nucl. Phys. B 348 (1991) 619; R. Guida and N. Magnoli, Nucl. Phys. B 471 (1996) 361.

[9] M. Caselle, P. Grinza and N. Magnoli, J. Stat. Mech. 0611 (2006) P003.

[10] M. Luscher, G. Munster and P. Weisz, Nucl. Phys. B 180 (1981) 1.

[11] M. Billò and M. Caselle, JHEP 0507 (2005) 038; M. Billò, M. Caselle and L. Ferro, JHEP 0602 (2006) 070.

[12] M. Caselle, F. Gliozzi, U. Magnea and S. Vinti, Nucl. Phys. B 460 (1996) 397.

[13] A. Allais and M. Caselle, JHEP 0901, 073 (2009).

[14] M. Caselle, P. Giudice, F. Gliozzi, P. Grinza and S. Lottini, JHEP 0711 (2007) 075.

[15] P. Giudice, F. Gliozzi, S. Lottini, JHEP 05 (2007) 010.

[16] R. J. Baxter, Exactly solved models in statistical mechanics (Academic Press, New York, 1982).

[17] A. B. Zamolodchikov and Al. B. Zamolodchikov, Ann. Phys. 120 (1979) 253; G. Delfino, Phys. Lett. B 450 (1999) 196; G. Delfino and P. Grinza, Nucl. Phys. B 682 (2004) 521.

[18] Ph. de Forcrand, O. Jahn, Nucl. Phys. 129 (Proc. Suppl.) (2004) 709. 